\title{
Change We Can Believe In: Structural and Content Dynamics within Belief Networks*
}

\author{
Nic Fishman ${ }^{\dagger}$ \\ Nicholas T. Davis
}

November 16, 2020

Running head: Belief dynamics

Keywords: Ideology, belief networks, network analysis

\footnotetext{
*The authors thank Jon Green, Mark Brandt, Chris Federico, Meredith Conroy, Laura Bucci, Kevin Reuning, Nathan Kalmoe, Dan McFarland, and Dave Peterson for input, kind words, and advice at various stages of this project. Three referees contributed excellent feedback that strengthened the final manuscript, and the authors are grateful to the editors for supplying helpful guidance during the revision process. No external funding supported this research; any remaining errors are our own.

${ }^{\dagger}$ Nic Fishman is an undergraduate student in the Department of Computer Science, Stanford University (njwfish@stanford.edu).

${ }^{\ddagger}$ Nicholas T. Davis is an Assistant Professor, Department of Political Science, University of Alabama (ntdavis2@ua.edu)
} 


\title{
Change We Can Believe In: Structural and Content Dynamics within Belief Networks
}

\begin{abstract}
Scholars have used network analysis to explore the structural properties of mass ideology. This manuscript incorporates two important, though ignored features in past research by investigating how time shapes the properties of belief networks for different populations of people those who exhibit high and low levels of political knowledge. We find that: (1) belief network density increases asymmetrically among people with high relative to low knowledge; (2) symbolic preferences are more central to belief networks irrespective of survey timing or population; in contrast, policy beliefs exhibit some increase in centrality over time among the politically knowledgeable; and, (3) a belief's centrality is unrelated to the amount of change it explains in other beliefs. Troublingly, this latter finding presents problems for describing belief networks using the vernacular of Conversian belief systems - a disconnect that seems grounded in the mismatch between Converse's individual-level theory and network analysis' population-based properties.
\end{abstract}

Word count (inclusive full text, figures, and references): 9.938 words

Replication materials: The data and materials required to verify the computational reproducibility of the results, procedures and analyses in this article are available on the American Journal of Political Science Dataverse within the Harvard Dataverse Network, at: https://doi.org/10.7910/DVN/ED5O5U. 
How do most people live without any thought? There are many people in the world, - you must have noticed them in the street, - how do they live? How do they get strength to put on their clothes in the morning?"

Emily Dickinson

Democracy requires that citizens navigate complex social and political choices. Although classic democratic theory took for granted the capacity of ordinary people to think logically and make rational political decisions (e.g. Downs 1957), analyses of Americans' political attitudes turn up an alarming amount of illogic and incongruity (Converse 1964; Achen and Bartels 2016; Kinder and Kalmoe 2017). Yet, while ordinary citizens may not think "ideologically," their belief systems nevertheless exhibit some amount of structure. An increasingly common way of visualizing the permutations these preferences take involves network analysis, which models how attitudes cohere in relation to each other and borrows heavily from Conversian ideas about the arrangement of such preferences (e.g. Boutyline and Vaisey 2017; Brandt, Sibley, and Osborne 2019).

Although past research has shown that network analysis can be adapted productively to the study of political ideology, we identify two important aspects of the survey response that have not been sufficiently explored within this framework. First, it is unclear whether belief networks are more well-developed among persons who know something about politics. If it is true that the "quality" of ideology varies by political acumen (Kalmoe 2020), then the properties of belief networks may similarly vary according to the extent to which people understand the political system. Second, the role of time in the development of political belief networks is unclear. Past research has yet to assess whether or how the content within and the structure of belief networks shift over time.

This uncertainty about the stability of belief networks over time and across groups raises several important questions. Conditional on knowledge, do citizens' belief networks exhibit structural changes in density over the course of an election? A large literature on campaign learning 
implies that beliefs are primed, if not changed (Jacobson 2015). Belief networks, then, might exhibit greater richness in connectivity over the course of an election if they function as "Conversian" representations of belief systems. By extension, if network structure is dynamic, then do the elements that comprise belief networks shift to and from the periphery? They should according to Converse's logic about the centrality of groups. Finally, answering these question raises another, perhaps more fundamental one: what does centrality within the belief network mean substantively? Does centrality predict which elements "constrain" individuals' political preferences? If not, then it seems unlikely that belief network analysis maps conceptually onto how political scientists have conventionally stylized belief systems. 1

This manuscript addresses these questions using panel data collected over the course of the 2008 presidential election. Several findings offer insight into the dynamics of belief networks. First, belief network density increases over time among our population of politically knowledgeable persons, which emphasizes how the informational richness of elections might shape belief network structure asymmetrically. Second, symbolic preferences are more central to belief networks irrespective of time or sub-population. Policy preferences, however, exhibit some increase in centrality over time, but only among the knowledgeable - illustrating, again, an important asymmetry that corroborates the idea that low-information publics may struggle to glean much from informational signals (Price and Zaller 1993; Arceneaux and Vander Wielen 2017; Bakker, Llekes, and Malka 2020). Third, we find that a belief's centrality is unrelated to the amount of change it explains in other beliefs. In other words, centrality, which ostensibly conveys an element's "importance" within the network analysis framework, does not resemble predictiveness, or the conventional measure of importance within standard parametric models.

On balance, these findings not only reveal the role that elections play in shaping the structure, content, and expression of political beliefs, but also raise questions regarding how to interpret the qualities of belief networks in relation to "ideology." This latter finding, in particular, presents

1. Hereafter, when we reference belief systems, we mean the theoretical configuration of preferences in the Conversian framework. Belief network, however, implies something different. This term embodies an artificial structure of preferences generated from network analysis. These things could, in theory, be quite analogous, but that is an open question to which we dedicate this paper. 
problems for describing belief networks using the vernacular of Conversian belief systems - a disconnect that seems grounded in the mismatch between Converse's individual-level theory and network analysis' population-based properties.

\section{Political ideology as a belief network}

The average person possesses a range of beliefs, values, and orientations that motivate their interaction with the political world (Jost 2006; Federico 2015). The shape that the combination of these preferences takes embodies the belief system, or what Converse (1964,pg. 3) described as a "configuration of ideas and attitudes in which the elements are bound together by some form of constraint or functional interdependence." While this definition seems straightforward, research on the structure or quality of political ideology has nevertheless struggled with both terminological precision and the evidentiary standards regarding what qualifies as "ideology" (e.g. Gerring 1997; Martin 1999; Jost 2006; Kinder and Kalmoe 2017; Azevedo et al. 2019).

Much of these debates involve disagreement over survey instrumentation, measurement, and analytical assumptions. However, an alternative way of thinking about the relationships among political preferences involves analytical tools that take the interdependence among individuals' attitudes seriously. In particular, network analysis provides such a framework by modeling a range of preferences as a network of "nodes" (belief elements) that are connected via "edges" (interdependent links). Unlike classic spatial models of ideology, which focus primarily upon latent structural models and the reduction of dimensionality, this modeling approach makes few assumptions about how political belief elements "ought" to relate to one another (e.g. Boutyline and Vaisey 2017). Central preferences form the locus or core of the network, which bind together other belief elements at the periphery. These relationships are formally embodied in the concept of "centrality," which reflects the positioning or importance of a given idea element relative to other preferences (c.f. Borgatti 2005).

Presumably, these properties of belief network analysis provide "high fidelity" (Baldassarri 
and Goldberg 2014, pg. 81) to Converse's ideas, in no small part because centrality seems to embody his ideas about the relative positioning and functionality of belief elements within the system. Yet, because network analysis fits a model over a population, it functionally models the "average" nature of the interrelationships among all idea elements in the aggregate. This disjuncture between levels of analysis raises questions about whether belief network analysis actually conforms to Converse's conceptualization of the belief system - questions that can only be answered by considering the dynamics of belief network qualities.

\section{Political campaigns and the temporal dynamics of belief net- works}

While network analysis has opened up tempting paths for investigating how mass beliefs are configured, much of the current psychological research involving political belief networks involves cross-sectional data (e.g. Boutyline and Vaisey 2017; Brandt et al. 2019). As a result, several questions involving the temporal stability of belief networks persist. First, it is not clear whether belief networks exhibit stationarity (i.e. their properties are constant over time) or if they change dynamically over the course of, say, an election. For example, while symbolic and operational elements exhibit different levels of centrality (Brandt et al. 2019), it is unclear whether this content shifts differently within the belief network over time. These dynamics are important because observing higher levels of policy centrality near an election, for example, would illustrate a responsiveness that is often discounted in minimalist depictions of ideology (e.g. Converse 2000; Kinder and Kalmoe 2017).

Second, a central premise in Converse's work involves the idea that "central" idea elements at the core of a belief system should bring other preferences into alignment. However, it is not clear whether or not network centrality has any "causal" bearing on content or vice versa. This ambiguity makes it difficult to unpack what centrality actually means for the belief network, which has important implications for assessing whether belief network analysis actually produces as an 
approximation of a Conversian belief system.

A political campaign is a useful context in which to explore these temporal dynamics for several reasons. Although mid-century conventional wisdom suggested that the effects of campaigns on citizens' preferences were minimal (e.g. Berelson, Lazarsfeld, and McPhee 1954), this view is now a minority one (Iyengar and Simon 2000; see Jacobson [2015] for a thorough review). Because campaigns are information-rich environments, they serve multiple purposes. They inform citizens about who candidates are (Alvarez 1998), what they stand for (Popkin 1991), and their qualifications (Johnston et al. 2004), which helps calibrate voters' personal beliefs (Bartels 1993) and, ultimately, their vote choice (Vavreck 2009; Sides, Tesler, and Vavreck 2019). In fact, debate about the role that campaigns play in changing beliefs notwithstanding, there is widespread agreement that campaigns achieve these outcomes by priming the salience of both political identities (Berelson et al. 1954; Erikson and Wlezien 2012; Huddy, Mason, and Aarøe 2015) and ideology (Gelman and King 1993; Hillygus and Shields 2008). As a result, comparing respondents' belief networks at the beginning and conclusion of an electoral campaign provides an excellent opportunity to not only explore the dynamics of political belief networks, but to analyze whether elections teach prospective voters "what goes with what."

\section{The importance of political knowledge}

However, the informational richness of campaigns notwithstanding, cue uptake is not distributed evenly among the mass public. As far back as the 1970s, scholars suspected that information supplied by elites generated inequalities in both knowledge and capacity. Thus, a natural "knowledge gap" might manifest where citizens of higher socioeconomic status would acquire information at a faster rate than peers of lower socioeconomic status (Tichenor, Donohue, and Olien 1970), in part because the political system exacerbates inequality. Citizens who perceive that the system does not "work" for them are less likely to be interested in navigating politics (Du Bois 1920; Delli Carpini and Keeter 1996). However, while it is plausible that the already information-affluent would become richer during an election (Price and Zaller 1993; Luskin, Fishkin, and Jowell 2002), the costs to acquire information are tied directly to the availability and diffusion of information. In turn, it is 
possible that the increased intensity and salience of political information found during a campaign may instead assist the otherwise-unaware in developing their attitudes. This idea corresponds to Zaller's (1992) proposition that "less sophisticated" voters may develop and become more confident in their political preferences over the course of a campaign, which serves up the type of informational signals that such citizens do not routinely see.

We take the possibility of such "heterogeneous" learning effects within political networks seriously because ideology constitutes different things for people who are (un)familiar with politics. In particular, political knowledge - sometimes couched as "awareness" or "sophistication" describes the ability of respondents to understand how politics "works." 2 Much research indicates that this acumen reliably shapes how values and beliefs are folded into and expressed within belief systems (e.g. Goren 2012; Miller, Saunders, and Farhart 2015; Kalmoe 2020; Federico and Malka 2018).

However, while knowledge reliably conditions ideology, the empirical evidence that campaigns may affect (un)aware citizens in different ways is more nuanced. Some scholars find that campaigns assist the least politically-aware; others find that citizens with higher levels of awareness are more responsive to these events (see: Claassen [2011] for a succinct review). Although most of this research program involves whether and how campaigns persuade, Arceneaux's (2006) research is particularly relevant here because it demonstrates how campaigns can link ideology to partisan preferences via priming. Conditional on knowledge, symbolic and instrumental preferences behave differently as the election nears, where "[1]ess politically sophisticated individuals use campaigns to learn basic features of the electoral landscape, such as defining which party matches them ideologically, whereas political sophisticates enter the campaign with a sense of the parties' ideologies relative to their own, and use campaigns to glean other types of electorally relevant considerations" (pg. 167).

We believe that this finding offers suggestive evidence of and an important justification for parsing belief network analysis by political knowledge for two reasons. First, as we show in the

2. We treat "sophistication" and "knowledge" as rhetorically equivalent for the purposes of this paper, but prefer the latter term due to its lack of elitist connotations. 
online supporting materials, individual-level differences in both political interest and news consumption vary among the more and less knowledgeable $\mathrm{S}^{3}$ Second, political knowledge conveys familiarity with the political system and its actors. The combination of both exposure to and individual motivation for interacting with the campaign environment should translate into different patterns of network development over time $4^{4}$ Because political knowledge is an effective summary property that distinguishes the politically (dis)interested and (un)aware, we expect to observe different content and structural changes in belief networks across these two groups over time 5

\section{Theoretical expectations}

Drawing on these ideas, we now outline the logic of several analyses that involve parsing the dynamics of belief network change among high- and low-knowledge citizens over the course of the 2008 presidential election.

\section{Changes in political belief network density}

We begin with a summary property of belief network structure - density - which permits us to test how the number of associations or connections among idea elements change over time. Because high-knowledge citizens understand politics, we expect that their belief network should be denser than low-knowledge respondents before a political campaign. Given that these individuals have a firmer grasp on politics and are more likely to navigate information-rich sources (Marshall 2019), it is possible that the density of their belief network will be constrained by ceiling effects (i.e. the belief network begins with more density and so has little room to grow). If so, then the density of this network might shift only nominally over the campaign.

In turn, if low-knowledge citizens express only a modest understanding of political facts and political coalitions, then they should begin a political campaign with a loosely connected belief

3. See Figures B1 and B2 on pages 7 and 8.

4. More generally, there is a wider literature on cue-taking that suggests such differences may arise in how information is processed. Arceneaux and Vander Wielen [2017], for example, find evidence of a positive relationship between sophistication and cue up-take; Bakker, Llekes and Malka [2020] demonstrate that informational cues are "best" used by persons with strong rather than weak identities, and political knowledge covaries with these preferences.

5. To be clear, the aggregate nature of network analysis makes establishing the underlying causal mechanism for such shifts more difficult than conventional individual-level analysis. While we believe the campaign-learning story is plausible, belief network shifts are likely not monocausal and future research on this question is encouraged. 
network (i.e. one that exhibits low density). As the campaign progresses, these persons should be exposed - either directly or indirectly - to political information that they might not otherwise receive during non-election periods. As such, we expect significant changes in belief network density for these individuals, where the number of associations among belief elements increase over time.

\section{Changes in the centrality of symbolic and operational content}

While density is an informative property within the belief network framework, centrality conveys the relative embeddedness of individual belief elements within a network. Thus, it is valued given our interest in the interconnectedness of belief elements. Although more appropriately described as a family of measures, recent political psyschology research usually explores one of three centrality types (e.g. Brandt, Sibley, and Osborne 2019; Brandt 2020): (1) strength centrality, a measure of the connectivity of a given node to other neighboring elements, which conveys the general importance of an element; (2) betweenness centrality, a measure of how much information passes through a belief element, which conveys whether a belief is a "bridge" to other preferences; and, (3) closeness centrality, which conveys the locational importance of an element, or the extent to which a belief element can influence the network quickly. $]^{6}$

Generally, when centrality is high (low) an instrument is presumed to be more (less) "important" to the network. However, as discussed previously, not all idea elements carry the same "weight." A major focus within research on ideology involves parsing symbolic preferences affective attachments toward political objects - from operational ones - the instrumental policy attitudes people hold (e.g. Ellis and Stimson 2012). This distinction is nontrivial. Research demonstrates that both partisanship (Greene 2004; Huddy, Mason, and Aaore 2015) and liberalconservative self-placement function as forms of social identity (Devine 2015). That is, individuals conceptualize their political orientations relationally, assigning positive (negative) emotional value to the in-group (out-group). In contrast, policy attitudes are conceptualized as instrumental pref-

6. This family of measures presumably reveals different relationships among nodes within the overarching belief network; however, as canonical network analysis texts detail, there are a great many centrality forms from which one could choose that might shape the conclusions one draws from network analysis (c.f. Borgatti 2005; Freeman 1978) 
erences. They are a set of cognitive beliefs about the appropriate strategies for governing social, economic, and political institutions..$^{7}$

To the extent that these elements are situated differently within belief systems, Converse (1964, pg. 46) himself noted that "the party and affect toward it are more central within the the political belief systems of the mass public than are the policy ends that the parties are designed to pursue.” Indeed, Brandt et al. (2019) find this proposition to be true. Symbolic belief elements are a great deal more central within a belief network than operational ones, suggesting that affective elements help bind belief networks together.

It is unclear, however, whether and how the properties associated with these belief elements might shift within the network over time. Given the fixity of identity (Niemi and Jennings 1991; Johnston 2006), it is plausible that symbolic content will resist changes in centrality over time, but it seems equally likely that such preferences could become more central within the belief network over the course of the election because campaigns prime group affect (Huddy, Mason, and Aaore 2015). Correspondingly, it is possible that instrumental policy preferences will exhibit a larger increase in centrality over the course of the election, in part because these idea elements are the currency of political campaigns, but also because it is less likely that these idea elements are interconnected when politics is less salient. In that case, the cascade of political signals that occur in the information environment as the election nears may increase the centrality of operational idea elements because their "floor" is low at the start of the campaign.

In either case, knowledge likely constrains how these elements change over time, although we have weak priors about what such changes might encompass. For example, because highknowledge citizens are more familiar with political matters, they may exhibit larger increases in centrality among operational preferences compared to low-knowledge persons. Alternatively, perhaps symbolic elements become more central among low-knowledge persons because they rely

7. Of course, this stylized distinction pitting "affective" against "cognitive" elements risks diminishing the notion that identities subsume policy preferences (i.e. identities are laden with some policy content) and that policy preferences are often encoded with affect (e.g. abortion attitudes [Turell et al. 1990] and immigration [Verkuyten 2004] elicit significant emotional responses from individuals). Nevertheless, the two sets of preferences are distinct enough to warrant some sort of separation (e.g. Ellis and Stimson 2012), artificial as it may be. 
more heavily on affective group attachments to navigate political space.

\section{The implications of centrality}

Finally, turning full circle, we investigate the substantive implications of centrality. If belief networks approximate Conversian belief systems, then we would expect content changes in beliefs to be closely associated with the structural properties of the network. Indeed, Converse's (1964, pg. 45) framework seems to rest on the idea that temporal stability of a belief is strongly related to its locational placement within the belief system (i.e. centrality). Yet, it is tricky to assess whether belief network centrality functions as Converse conceived it, in part because Converse's expectations about centrality involved how belief elements function over time.

In practice, centrality is often stylized as a causal property. Indeed: “...just as any theory of belief system dynamics must include connections between elements, these connections must be causal in some way. That is, one element will influence connected elements" (Brandt and Sleegers 2020, pg. 4). The correspondence between belief system and belief network centrality, however, has been taken mostly at face value within the study of ideology-as-belief-network (Boutyline and Vaisey 2017; Brandt et al. 2019; Bringman et al. 2019). Yet, Dablander and Hinne (2019, pg. 2) caution that centrality "is easily confused with having causal influence. However, this does not follow from the network paradigm... as it provides only a statistical representation of the underlying system, not a causal one."

In fact, the "causal" nature of centrality in belief networks - that is, whether more central beliefs affect other belief elements in a predictive capacity, for example - is an open empirical question. To assess whether centrality has a "causal" nature, we can explore how it is related to more conventional estimates of "importance" within regression frameworks. As a rough proxy for the quantity we really want but cannot test - the causal influence of beliefs on network structure over time - we propose to substitute a kind of "predictiveness" heuristic that functions as a measure of instrumental importance.

If voters have a set of largely unordered beliefs at the start of the election (plausibly because they are not that entrenched in politics), then exposure to a campaign's information environment 
may alter their beliefs in accordance with the predominating frames used by elites. If there are some "central" beliefs that voters are less willing to change their minds about, then we would expect those to pull and push their other opinions over time. In that case, beliefs at the start of the election that are more central ought to influence whether other beliefs change over the course of the election. Although we formalize this logic mathematically below, our specific expectation can be stated as follows: if belief network centrality roughly approximates Conversian centrality - as prevailing theories of belief networks presume - then we should observe that belief centrality constrains downstream beliefs. In other words, central beliefs should, correspondingly, be "predictive" ones. Whether this relationship is conditional on knowledge, as we speculate in the prior sections, is an open question.

\section{Data and design}

The forthcoming analysis uses data from the 2008-2009 American National Election Studies Panel Study at Waves 1 (January, 2008) and 10 (October, 2008). 701 respondents completed surveys at both waves. The specific waves were selected due to the overlap of available instruments common to both questionnaires. 8

\section{Measures}

Political preferences. Our choice of belief elements to model is limited by the survey design. Policy questions and political identification are rarely asked at common waves. Thus, our pool of available items is constrained to all of those questions that were included on the Wave 1 and 10 surveys, which still included a wide spectrum of policy areas. The six instruments included: attitudes toward gay marriage, taxes, single payer healthcare, habeas rights for suspected terrorists, court orders for wiretapping, and illegal immigration. For each policy item, respondents were asked whether they favored (opposed) a stated policy solution. They then received a branching question that asked whether they favored (opposed) an option "a little," "moderately," or "a great

8. Although attrition in panel surveys is a problem (the individuals who do not participate at Wave 10 may be systematically different from persons who completed both surveys), the dearth of panel data that includes a common battery of identity and issue questions at multiple time points means there are few alternatives to this dataset. 
deal." This sequence generated a response set for each item that ranged from 1 to 7 , where low (high) values correspond to traditionally left-leaning (right-leaning) preferences.

We use four items that tap into symbolic preferences. Two items asked whether respondents liked (disliked) the parties and employed similar branching question prompts to establish the strength of affect felt toward the Republican and Democratic Parties. In addition, both the traditional liberal-conservative and party identification instruments were included. Again, all measures ranged from 1 (left-leaning) to 7 (right-leaning). 9

Political knowledge. To stratify the sample on the basis of political acumen, we relied on six items that assess factual political knowledge. Respondents were asked 1) the number of times U.S. Presidents can be elected, 2) the number of years that U.S. senators serve, 3) the number of senators from each state, 4) the number of years members of the U.S. House of Representatives serve, 5) the successor to the Vice President, and 6) the necessary percentage of votes needed to override a presidential veto. Correct answers were coded 1 and otherwise 0 . We then calculate an additive scale. High-knowledge persons answered at least five of six questions correctly $(\mathrm{n}=242)$; respondents in the low-knowledge group answered four or fewer items correctly $(n=459)$.

\section{Method}

Epskamp et al. (2018) document an analytical approach that creates a network of survey responses using pairwise Markov random fields (PMRF), where the network is comprised of nodes connected by undirected edges indicating conditional dependence between two variables. In our case, we assume the joint is distributed as a multivariate normal, which permits a Gaussian graphical model where the edges are treated as partial correlation coefficients that are estimated using the covariance matrix of the data or polychoric correlations in the case of ordinal data (as most political survey data is). This structure accounts for the dependence/independence relations in the data, affording clear node relationships and intuitive structural properties (e.g. the stronger an edge is between two nodes, the more related those node are net of all other positions).

9. We restrict symbolic elements to these items, which means excluding candidate affect. Unfortunately, the coverage of these instruments for a broad array of figures across waves is poor. 
Of primary interest here are comparisons of several belief networks (e.g. networks using data collected at different waves) constructed on several populations (e.g. persons who possess different levels of political knowledge). Boutyline and Vaisey (2017) recommend approaches for comparing sub-populations, primarily along two dimensions involving whether the logics underlying network structure and / or the degree of network organization differs. ${ }^{10}$ Drawing on this work, we propose to compare sets of belief networks by generating the belief networks for two populations (stratified by knowledge) at two time points (January, October 2008); we then use non-parametric permutation tests to evaluate the significance of differences in overall density and strength centrality. Tests of this type entail repeatedly randomizing the assignment of individuals between the two populations under examination, re-constructing the belief networks, and aggregating the difference of the property of interest into a distribution of differences. The distribution generated in this fashion can be used to perform a two-tailed t-test on the observed difference, which reveals whether changes in network properties (and between sub-populations) are significant. 11

\section{Results}

\section{Network density over time}

We begin by generating four belief networks in Fig. 1 that constitute the partial correlations among nodes for high- and low-knowledge persons at Waves 1 and 10. Holding the node positions constant, it appears that high-knowledge persons in Wave 10 (Panel D) possess stronger associations among the idea elements within their belief network relative to the other networks presented in Panels A - C (i.e. the connections [lines] are thicker than those observed in other panels). This quality is important because it serves as a heuristic for the overall amount of structure or amount of dependence in a given network, which describes how "organized" a population's beliefs are.

10. Here, "logics" refer to the direction of the correlation, positive or negative; two networks have the same underlying logic if all the correlations are directed in the same way.

11. Several R packages were useful in this task: Canty and Ripley's (2020) boot allowed for convenient bootstrapping for permutation tests; Epskamp and Fried's (2020) package bootnet was used to construct the belief networks; Epskamp, Giulio, and Haslbeck's (2020) qgraph, as well as Harrell Jr.'s (2019) Hmisc were vital in creating graphical representations of the networks. 
We calculate and compare densities for the four networks in Table 1. Several results are notable. First, it is curious that the difference in the overall densities between high- and lowknowledge persons, which is modest, is not significantly different at Wave 1 (the p-value narrowly misses the more liberal $p<0.10$ ). To the extent that the information environment is only modestly saturated with political signals during the early months of the campaign, it would seem that the tendrils connecting belief elements in their "fallow" state do not differ across high- and lowknowledge populations. This finding deviates from our assumption that high- and low-knowledge persons ought to naturally differ in the structure of their belief networks, irrespective of time.

However, changes in density over time are conditional upon knowledge. Over the course of the campaign, the network for low-knowledge persons does not become significantly denser. The amount of organization among beliefs is effectively static. In contrast, high-knowledge respondents do see significant increases in network density. Among this population of persons, political preferences become significantly more well-connected. This pattern seems to match Converse's (1964, pg. 44) prediction that "[t]he configuration of political beliefs held by individuals simply becomes increasingly idiosyncratic as we move to less sophisticated people." To the extent that denser belief networks are less idiosyncratic, that seems to be the case here.

\begin{tabular}{clc} 
Networks Compared & Difference in Density & \\
\hline \hline Low-knowledge W10 vs W1 & 0.0239 & $(p=0.314)$ \\
High-knowledge W10 vs W1 & 0.2038 & $(p=0.01)^{* *}$ \\
Wave 1 High-knowledge vs Low-knowledge & 0.0573 & $(p=0.106)$ \\
Wave 10 High-knowledge vs Low-knowledge & 0.2372 & $(p<0.001)^{* * *}$ \\
\hline
\end{tabular}

Table 1: Differences in the overall density of belief networks over time and between high- and lowknowledge respondents. Note: Density is calculated as the sum of the network edges. Given that we have aligned the underlying response scales to all match a liberal/conservative scale, positive edges map to "coherent" collections of beliefs within the current party-policy millieu. Taking the sum is a methodological necessity to avoid boundary conditions in our randomization tests. ${ }^{*} p<0.05,{ }^{* *} p<0.01,{ }^{* * *} p<0.001$

\section{The dynamics of operational and symbolic content}

Having assessed how the organizational properties of belief system change over time, we now explore the importance of symbolic and operational content within these networks. Here, we are 
concerned with whether these idea elements are 1) more or less central at each wave for each group, and 2) whether the strength centrality of such elements shift over the course of the election. Again, we generate visual representations of theses changes, which are displayed in Fig. $2 \mid$\begin{tabular}{|l|l|}
\hline 2 \\
\hline
\end{tabular}

We make several observations. First, among persons in the low-knowledge group and across both waves, symbolic elements are significantly more central than operational ones. In contrast, whereas symbolic elements were more central than operational ones among high-knowledge respondents at Wave 1, these differences disappear by Wave 10. In other words, this change in strength centrality is consistent with a story of high-knowledge persons developing more complex positions on operational beliefs as they (presumably) learn about them over the course of the campaign. This finding makes some sense from the standpoint that such "ideological-thinking" is often restricted to those people who are actually familiar with how politics works.

To investigate these findings further, we disaggregate the content contained within these categories to assess how the individual belief elements included in the networks shift in centrality over time. Fig. 3 portrays two series of illustrations. 13 First, Panels A and B portray Waves 1 and 10 strength centrality for each of the 10 belief element among low- and high-knowledge persons, respectively. Beginning first with Panel A, although we observed that neither symbolic nor operational elements shifted in centrality over the campaign for the low-knowledge group in Figure 2, we nonetheless find some significant differences in strength centrality across individual belief elements over time. Taxes, wire-tapping and healthcare became modestly more central by Wave 10, while habeas and immigration attitudes become less central. Further, while partisanship, ideological identification, and affect toward the Democratic Party were more central at Wave 10, there was a massive reduction in centrality in Republican Party affect at that wave.

Among the high-knowledge group in Panel B, we observe large changes in strength centrality

12. Readers interested in supplementary analysis involving betweenness and closeness centrality will find these models replicated in the online supporting materials, Section C, pages 11-13. Results for Panel A generalize across all three metrics; results for Panels B and $\mathrm{C}$ are common across strength and closeness centrality, but not betweenness centrality; results illustrated in Panel D also mostly generalize across all three metrics.

13. Supplementary analysis involving estimates for betweenness and closeness centrality of these individual elements can be found in the online supporting materials, Section C, pages 17-20. Here, there is some modest variation in the centrality of particular belief elements across types of centrality, but the broader patterns are similar. 
among three issues (tax, habeas, and same-sex marriage preferences), which seems to have driven the shift in operational content over time in the prior figure. In contrast to these items, we observe no meaningful changes in the centrality of symbolic instruments. Centrality among these items begins the election "high" and remains relatively stable over the duration of the election.

Moving to the bottom two panels, we contrast belief element strength centrality for the two groups against each other at Wave 1 and Wave 10. In Panel C, we observe large differences between high- and low-knowledge respondents on tax preferences and liberal-conservative self-placement and more modest differences on several other issues. Democratic Party affect among low-knowledge persons also appears more central than among those with higher levels of knowledge at Wave 1. Pivoting to Wave 10 in Panel D, we observe a somewhat similar picture. Whereas persons with greater political knowledge exhibit much more strength centrality on liberal-conservative self-placement, taxes, and gay marriage, low-knowledge respondents' partisanship exhibits slightly higher levels of strength centrality.

To some extent, these findings are straightforward. Ideology means something qualitatively different for individuals who understand the political system. This notion is reflected in the growth of strength centrality in cultural (e.g. gay marriage) and economic preferences (e.g. taxation) among the more knowledgeable over time and again in the differences in strength centrality of these elements relative to low-knowledge persons in Wave 10. Further, the most prominent difference in strength centrality among the two groups' belief elements involves liberal-conservative selfidentification. Past research suggests that these labels are most productive to individuals who understand what these labels mean (c.f. Kinder and Kalmoe 2017), and this result corroborates that finding.

What are we to make, however, of the significant heterogeneity in strength centrality among the various operational elements? Although high-knowledge respondents observe a net increase in operational strength centrality from Waves 1 to 10 (per Fig. 2), this change seems primarily driven by several considerations including tax, habeas, and same-sex marriage preferences. In fact, these changes seem track with other survey data of issue importance in the 2008 election. Pew 
(2008) and Gallup (2008) found, for example, that voters accorded high levels of importance to the economy and foreign policy (which encompasses both immigration and War-on-Terror belief elements). Among Republican voters, moral issues were deemed important, while Democrats placed significant attention on healthcare 14 In turn, these issues were among those emphasized by the parties during the campaign (Vavrek 2009; Hillygus and Henderson 2010; Balz and Johnson 2009), which occurred against a shifting social, economic, and political backdrop that included a financial crisis, stagnating Iraq foreign policy, and shifting cultural mores. To some extent, we see these issues become more interconnected for knowledgeable persons nearer to the election.

\section{How "important" is centrality? Is it predictive?}

Building on the results of the previous section, we turn next to probing the meaning of strength centrality through the lens of time in order to better understand the substantive implications of centrality. We begin with a simple model for predicting changes over time in issue $B^{(k)}$ from $B^{(j)}$ :

$$
B_{i, 10}^{(k)}-B_{i, 1}^{(k)}=\alpha+\beta B_{i, 1}^{(j)}+\varepsilon_{i j k}
$$

Where $B_{i, w}^{(k)}$ is the position held by respondent $i$ on position $k$ at wave $w, \alpha$ is an intercept term, $\beta$ is the slope, and $\varepsilon_{i j k}$ is an independent error. Then, using the $R^{2}$ of this model we can define the predictiveness of an issue $B^{(j)}$ on another issue $B^{(k)}$ :

$$
R_{j k}^{2}=1-\frac{\sum_{i}\left(B_{i, 10}^{(k)}-B_{i, 1}^{(k)}-\hat{\alpha}-\hat{\beta} B_{i, 1}^{(j)}\right)^{2}}{\sum_{i}\left(B_{i, 10}^{(k)}-B_{i, 1}^{(k)}-\frac{1}{N} \sum_{i}\left(B_{i, 10}^{(k)}-B_{i, 1}^{(k)}\right)\right)^{2}}
$$

In turn, we can develop a general metric for the predictiveness of the issue $B^{(j)}$ :

$$
P_{j}=\sum_{k \neq j} R_{j k}
$$

Our predictiveness metric, $P_{j}$, will be high if the issue $B^{(j)}$ is an issue important to structuring

14. Given that partisans ranked issue importance differently (Pew 2008), future analysis of partisan differences in centrality may be productive. 
voters' other beliefs ${ }^{15}$ It is this estimate that we will link to centrality $\left(C_{j}\right)$ in several ways, which are all rooted in the longstanding notion that centrality approximates predictiveness.

Although scholars generally assume that the beliefs that emerge as more central in belief network analysis are in fact more important in the framing of new information or in the pulling and pushing of other beliefs, it is not clear whether this supposition is correct. If it is, then the most central belief elements at Wave 1 should also have the highest predictiveness. Because stability and importance are proposed to be interlinked in the conventional depiction of centrality (e.g. Brandt, Sibley and Osborne 2019, pg. 1361), upstream centrality should logically constrain downstream predictiveness. We can call this the "prospective" relationship, and we give our specification for this model in (4). Alternatively, we might expect that elements that appear to have structured the belief network over the course of the election would be more central at Wave 10; we call this the "retrospective" relationship, and give the specification in (5). Finally we offer an alternate specification for this retrospective relationship where the elements that appear to have structured the belief network over the course of the election would have experienced the most (positive) change in centrality over the course of the election, see (6).

$$
\begin{aligned}
P_{j} & =\alpha_{\text {prospective }}+\beta_{\text {prospective }} C_{j, 1}+\varepsilon_{j} \\
C_{j, 10} & =\alpha_{\text {retrospective }}+\beta_{\text {retrospective }} P_{j}+\varepsilon_{j} \\
C_{j, 10}-C_{j, 1} & =\alpha_{\text {change }}+\beta_{\text {change }} P_{j}+\varepsilon_{j}
\end{aligned}
$$

Figure 4 illustrates the results of these different ways of thinking about the relationship between predictiveness and centrality, conditional upon knowledge. First, are elements that are central at Wave 1 also structuring elements (i.e. elements that have predictive significance)? This is the "prospective" model, most consistent with the causal theories we give above. The estimates

15. It is relevant to note that this will be a very aggressive estimate of the relationship among variables. An implicit assumption of this model is that the beliefs at Wave 1 are all that influences belief changes at Wave 10, which is a strong, if not flawed assumption. But, this minimal toy model should be sufficient for the analyses in which we are interested. If we cannot recover the expected pattern of findings using this approach, then the inclusion of stronger modeling assumptions (i.e. controls) should only make the task more difficult. 


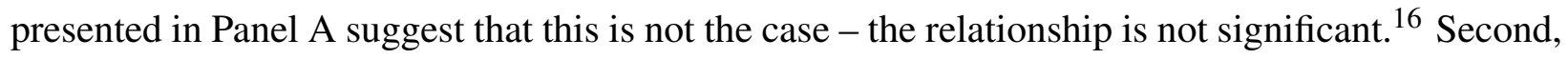
are elements that are central at Wave 10 structuring elements? This would be the "centrality as retrospective causality" argument; maybe beliefs that were structuring (i.e. highly-predictive) at Wave 1 are more central in the resultant belief network at Wave 10. In Fig. 4 Panel B we again observe a non-significant relationship; the slopes are essentially flat. We conclude with a tweak to this analysis: are elements that move the most from the periphery to the center from Wave 1 to Wave 10 structuring elements? This would be consistent with predictiveness explaining the change in centrality, but, again, in Fig. 4 Panel C, we find a non-significant relationship (and one that is pointed in the wrong direction) 17

What are we to make of these results? Technically, centrality captures both whether a belief is structuring another belief and whether a belief is structured by another belief. Thus, it fails to discriminate between the two qualities, which have different substantive implications. These results do not mean that centrality is uninterpretable, but perhaps imply that it is capturing some generalized sense of belief element salience, rather than anything that resembles predictiveness. Central belief elements may be strongly interconnected with other preferences, but they do not exhibit the "constraining" behaviors that scholars would expect central beliefs to exhibit. This point makes some sense, however, when we consider the theoretical-analytical mismatch between the Conversian framework that scholars want belief network analysis to approximate and the aggregate nature of network analysis more generally - belief network analysis involves population, not individual-level analysis. In other words, is difficult to expect the results of a population-level analysis to approximate how Converse hypothesized an individual's mental processes operate.

16. Modeling output for these relationships is available in the online supporting materials, Section D, page 25 .

17. In Section D of the online supporting materials (pages 22-24), we replicate these analyses with betweenness and closeness centrality and find the same pattern of non-signifcant or counter-intuitive results. Irrespective of the centrality metric we use here, the relationship between predictiveness and centrality never manifests as we would expect. 


\section{Discussion and conclusion}

Presumably, new methodological techniques and powerful computing resources can provide tools to help unlock the mysteries regarding how ordinary people think about matters of politics. Nevertheless, ideology remains an elusive quantity. Fields' (1990) comments are perhaps an appropriate warning about the difficulty of subjecting ideology to analysis. She argues that ideology

...is not a material entity, a thing of any sort, that you can hand down like an old garment, pass on like a germ, spread like a rumour, or impose like a code of dress or etiquette. Nor is it a collection of disassociated beliefs - attitudes is the favored jargon among American social scientists and the historians they have mesmerizedthat you can extract from their context and measure by the current or retrospective survey research.

Whatever the essence of "ideology," it does not succumb easily to quantification. We ought to take seriously, then, the criticism that the complex nature of ideology resists stylized manifestations of it.

Still, this pessimism notwithstanding, quantifying the contours and dynamics of belief systems is an exercise with important implications for social and political outcomes. Identifying the conditions under which beliefs structure and are structured by other beliefs gives us a window into why individuals may receive or reject incorporating information about politics when navigating the campaign context. Understanding those dynamics are valuable to both practitioners and academics alike.

This paper contributes to this task by investigating the properties of belief networks to test whether they behave like Conversian belief systems. A central premise in the study of ideology involves the idea that well-developed mental frameworks should exhibit many connections among beliefs ${ }^{18}$ We would have expected that belief networks naturally become more dense as the information environment becomes richer - but perhaps only among those people for whom politics is familiar. In the case presented here, a temporal snapshot of voters' attitudes at either January

18. Certainly, the range of a system may be narrow or broad (per Converse), but, irrespective of the scope, a "welldeveloped" system ought to exhibit interconnectedness among elements. 
or October 2008 would have led to very different conclusions about this property. We found that density in the belief network was conditionally dynamic - among high-knowledge persons, it increased over the course of the election, while network density among individuals with lower levels of knowledge remained unchanged. This finding suggests that pooling across surveys for the purposes of network analysis may be problematic because the timing of data collection matters. By extension, this within-election dynamic suggests that across-election dynamics may be similarly relevant. The elements that structure the belief network in one election may or may not structure it in future ones with new candidates and different issue agendas.

Our second contribution was to scrutinize the property of (strength) centrality. Our interest in this concept stems from Converse's preoccupation with the relative positioning of idea elements within the belief system. We showed that strength centrality among operational and symbolic elements shifts differentially over time for both high- and low-knowledge people. Further, our analysis produced some surprising results - namely, it is not abundantly clear whether or not strength centrality actually resembles the sort of predictive importance that Converse ascribed to it.

These findings muddy how we ought to interpret the "importance" of centrality when modeling ideology as a belief network. ${ }^{19}$ As a result, it is worth pondering what the results derived from network analysis mean for the individual. Although network analysis does not allow for individual-level analysis, we were nevertheless able to parse belief networks by political knowledge, which is important given what scholars know about the relationship between the "quality" of ideology and such acumen (e.g. Kinder and Kalmoe 2017). To the extent that other demographic features might moderate relationships among idea elements, network analysis may obscure such contingencies.

In sum, while belief networks appear useful for stylizing ideology as an organizational framework, we suspect that they only weakly resemble the person-level "belief systems" of political psychology. In particular, the lack of a clear and sensible relationship between predictiveness and

19. Notwithstanding these conclusions, more research is needed. While we have demonstrated that the properties of belief networks are dynamic, it is less clear whether the structure of belief networks regress. That is, does centrality recede and content change as the information context becomes less rich? Unfortunately, the present dataset is limited and does not allow for such analyses. 
centrality uncovered here suggests that, while Converse's depiction of centrality is elegant, it nevertheless remains an elusive quality to quantify. The mismatch between the aggregate nature of network analysis and Converse's individual-level framework is probably partly to blame. It is difficult to imagine that centrality estimates fit across a population of people will correlate with the extent to which instruments predict individual-level belief changes. As a result, we would be hesitant to ascribe Conversian theory to the belief network analysis, attractive as that may be. However, if ideology emerges from social order, then trying to wrangle sensible structure out of discrete belief elements via belief network analysis may offer a window into the permutations that "ideology" manifests in the wider social-political environment. Brandt (2020), for example, analyzes belief networks cross-nationally and finds that certain institutional features help explain the permutations that belief networks take in different country contexts. In this vein, and to the extent that understanding the latent structure of such beliefs within the mass public may explain prevailing political cleavages and agendas, belief network analysis remains valuable. 


\section{References}

Achen, Christopher H, and Larry M Bartels. Democracy for realists: Why elections do not produce responsive government. Vol. 4. Princeton University Press, (2016). ISBN: 1400888743.

Alvarez, R Michael. Information and elections. University of Michigan Press, (1998).

American National Election Studies. American National Election Studies (ANES) Panel Study, 2008-2009. ICPSR29182-v1., (2009). http://doi.org/10.3886/ICPSR29182.v1.

Arceneaux, Kevin. "Do campaigns help voters learn? A cross-national analysis.” British Journal of Political Science 36, no. 1 (2006): 159-173.

Arceneaux, Kevin, and Ryan J Vander Wielen. Taming intuition: How reflection minimizes partisan reasoning and promotes democratic accountability. Cambridge University Press, (2017).

Azevedo, Flavio, John T Jost, Tobias Rothmund, and Joanna Sterling. "Neoliberal ideology and the justification of inequality in capitalist societies: Why social and economic dimensions of ideology are intertwined.” Journal of Social Issues 75, no. 1 (2019): 49-88.

Bakker, Bert, Yphtach Lelkes, and Ariel Malka. "Understanding Partisan Cue Receptivity: Tests of Predictions from the Bounded Rationality and Expressive Utility Perspectives." Journal of Politics July, no. 3 (2020): doi.org/10.1086/707616.

Baldassarri, Delia, and Amir Goldberg. "Neither ideologues nor agnostics: Alternative voters' belief system in an age of partisan politics." American Journal of Sociology 120, no. 1 (2014): 45-95.

Balz, Daniel J, and Haynes Bonner Johnson. The Battle for America, 2008: The Story o fan Extraordinary Election, (2009). 
Bartels, Larry M. “Messages received: The political impact of media exposure.” American political science review 87, no. 2 (1993): 267-285.

Berelson, Bernard R, Paul F Lazarsfeld, William N McPhee, and William N McPhee. Voting: A study of opinion formation in a presidential campaign. University of Chicago Press, (1954).

Borgatti, Stephen P. “Centrality and network flow.” Social networks 27, no. 1 (2005): 55-71.

Boutyline, Andrei, and Stephen Vaisey. "Belief network analysis: A relational approach to understanding the structure of attitudes." American Journal of Sociology 122, no. 5 (2017): 13711447.

Brandt, Mark J. "Estimating and examining the replicability of belief system networks." Collabra: Psychology 1, no. 24 (2020).

Brandt, Mark J, Chris G Sibley, and Danny Osborne. "What Is Central to Political Belief System Networks?" Personality and Social Psychology Bulletin 45, no. 9 (2019): 1352-1364.

Brandt, Mark J., and Willem W.A. Sleegers. "Evaluating Belief System Networks as a Theory of Political Belief System Dynamics," (2020).

Bringmann, Laura F, Timon Elmer, Sacha Epskamp, Robert W Krause, David Schoch, Marieke Wichers, Johanna TW Wigman, and Evelien Snippe. "What do centrality measures measure in psychological networks?" Journal of Abnormal Psychology 128, no. 8 (2019): 892.

Buckingham, Willis J. Emily Dickinson's Reception in the 1890s: A Documentary History. University of Pittsburgh Press, 1989.

Canty, Angelo, and Brian Ripley. Package 'boot'.

Claassen, Ryan L. "Political awareness and electoral campaigns: Maximum effects for minimum citizens?" Political Behavior 33, no. 2 (2011): 203-223. 
Converse, Philip E. "Assessing the capacity of mass electorates.” Annual review of political science 3, no. 1 (2000): 331-353.

—. "The nature of belief systems in mass publics." Critical Review 18) (1964): 1-74.

Dablander, Fabian, and Max Hinne. "Node centrality measures are a poor substitute for causal inference." Scientific reports 9, no. 1 (2019): 6846.

Delli Carpini, Michael X, and Scott Keeter. What Americans know about politics and why it matters. Yale University Press, (1996).

Devine, Christopher J. "Ideological social identity: Psychological attachment to ideological ingroups as a political phenomenon and a behavioral influence." Political Behavior 37, no. 3 (2015): 509-535.

Downs, Anthony. An Economic Theory of Democracy. New York: Harper, (1957).

Du Bois, William Edward Burghardt. Darkwater: Voices from within the Veil. Courier Corporation, (1920) 1999.

Ellis, Christopher, and James A Stimson. Ideology in America. Cambridge University Press, (2012).

Epskamp, Sacha, Denny Borsboom, and Eiko I Fried. "Estimating psychological networks and their accuracy: a tutorial paper." Behavior Research Methods 50, no. 1 (2018): 195-212.

Epskamp, Sacha, Giulio Costantini, and Jonas Haslbeck. "Package 'qgraph'."

Epskamp, Sacha, and Eiko I Fried. 'Package 'bootnet'," 2015.

Erikson, Robert S, and Christopher Wlezien. The timeline of presidential elections: How campaigns do (and do not) matter. University of Chicago Press, (2012). 
Federico, Christopher M. "The structure, foundations, and expression of ideology." In New directions in public opinion, edited by Adam Berinsky, 103-125. Routledge, (2015).

Federico, Christopher M, and Ariel Malka. "The contingent, contextual nature of the relationship between needs for security and certainty and political preferences: Evidence and implications." Political Psychology 39 (2018): 3-48.

Fields, Barbara Jeanne. "Slavery, race and ideology in the United States of America." New Left Review 181, no. 1 (1990): 95-118.

Freeman, Linton C. “Centrality in social networks conceptual clarification.” Social networks 1, no. 3 (1978): 215-239.

Gallup. “Obama Has Edge on Key Election Issues,” (2008). Accessed April 9, 2020. https://news. gallup.com/poll/108331/obama-has-edge-key-election-issues.aspx.

Gelman, Andrew, and Gary King. "Why are American presidential election campaign polls so variable when votes are so predictable?” British Journal of Political Science 23, no. 4 (1993): 409-451.

Gerring, John. “Ideology: A definitional analysis.” Political Research Quarterly 50, no. 4 (1997): 957-994.

Goren, Paul. "Political values and political awareness.” Critical Review 24, no. 4 (2012): 505-525.

Greene, Steven. "Social identity theory and party identification.” Social Science Quarterly 85, no. 1 (2004): 136-153.

Harrell Jr, Frank E. "Package 'Hmisc'."

Hillygus, D Sunshine, and Michael Henderson. "Political issues and the dynamics of vote choice in 2008." Journal of Elections, Public Opinion and Parties 20, no. 2 (2010): 241-269. 
Hillygus, D Sunshine, and Todd G Shields. The persuadable voter: Wedge issues in presidential campaigns. Princeton University Press, (2008).

Huddy, Leonie, Lilliana Mason, and Lene Aarøe. "Expressive partisanship: Campaign involvement, political emotion, and partisan identity." American Political Science Review 109, no. 1 (2015): 1-17.

Iyengar, Shanto, and Adam F Simon. "New perspectives and evidence on political communication and campaign effects." Annual review of psychology 51, no. 1 (2000): 149-169.

Jacobson, Gary C. "How do campaigns matter?” Annual Review of Political Science 18 (2015): $31-47$.

Johnston, Richard. "Party identification: Unmoved mover or sum of preferences?" Annu. Rev. Polit. Sci. 9 (2006): 329-351.

Johnston, Richard, Michael G Hagen, Kathleen Hall Jamieson, et al. The 2000 presidential election and the foundations of party politics. Cambridge University Press, (2004).

Jost, John T. "The end of the end of ideology." American psychologist 61, no. 7 (2006): 651-670.

Kalmoe, Nathan P. "Uses and abuses of ideology in political psychology." Political Psychology 41, no. 4 (2020): 771-793.

Kinder, Donald R, and Nathan P Kalmoe. Neither liberal nor conservative: Ideological innocence in the American public. University of Chicago Press, (2017).

Luskin, Robert C, James S Fishkin, and Roger Jowell. "Considered opinions: Deliberative polling in Britain.” British Journal of Political Science 32, no. 3 (2002): 455-487.

Marshall, John. "Signaling sophistication: How social expectations can increase political information acquisition.” The Journal of Politics 81, no. 1 (2019): 167-186. 
Martin, John Levi. "Entropic measures of belief system constraint.” Social Science Research 28, no. 1 (1999): 111-134.

Miller, Joanne M, Kyle L Saunders, and Christina E Farhart. "Conspiracy endorsement as motivated reasoning: The moderating roles of political knowledge and trust." American Journal of Political Science 60, no. 4 (2016): 824-844.

Motta, Matthew P. "What do interviewer intelligence ratings actually measure?" Research \& Politics 3, no. 3 (2016).

Nicholson, Stephen P. "Dominating cues and the limits of elite influence." The Journal of Politics 73, no. 4 (2011): 1165-1177.

Niemi, Richard G, and M Kent Jennings. "Issues and inheritance in the formation of party identification.” American Journal of Political Science, (1991), 970-988.

Pew. "Results from the 2008 Annual Religion and Public Life Survey," (2008). Accessed April 9, 2020. https://www.people-press.org/2008/08/21/section-3-issues-and-the-2008-election/.

Popkin, Samuel L. The reasoning voter: Communication and persuasion in presidential campaigns. University of Chicago Press, (1991).

Price, Vincent, and John Zaller. "Who gets the news? Alternative measures of news reception and their implications for research.” Public opinion quarterly 57, no. 2 (1993): 133-164.

Sides, John, Michael Tesler, and Lynn Vavreck. Identity crisis: The 2016 presidential campaign and the battle for the meaning of America. Princeton University Press, (2019).

Tichenor, Phillip J, George A Donohue, and Clarice N Olien. "Mass media flow and differential growth in knowledge.” Public opinion quarterly 34, no. 2 (1970): 159-170. 
Turell, Susan C, Mary W Armsworth, and John P Gaa. "Emotional response to abortion: a critical review of the literature." Women \& therapy 9, no. 4 (1990): 49-68.

Vavreck, Lynn. The message matters: The economy and presidential campaigns. Princeton University Press, (2009).

Verkuyten, Maykel. "Emotional reactions to and support for immigrant policies: Attributed responsibilities to categories of asylum seekers.” Social Justice Research 17, no. 3 (2004): 293314.

Zaller, John R. The nature and origins of mass opinion. Cambridge university press, (1992). ISBN: 0521407869. 


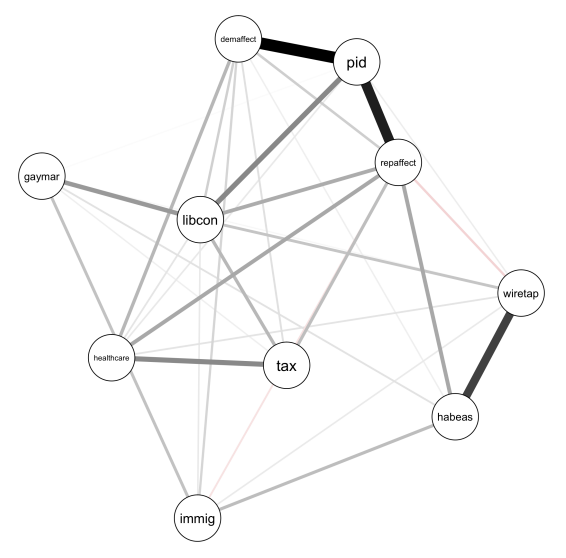

(a) Low-knowledge, Wave 1

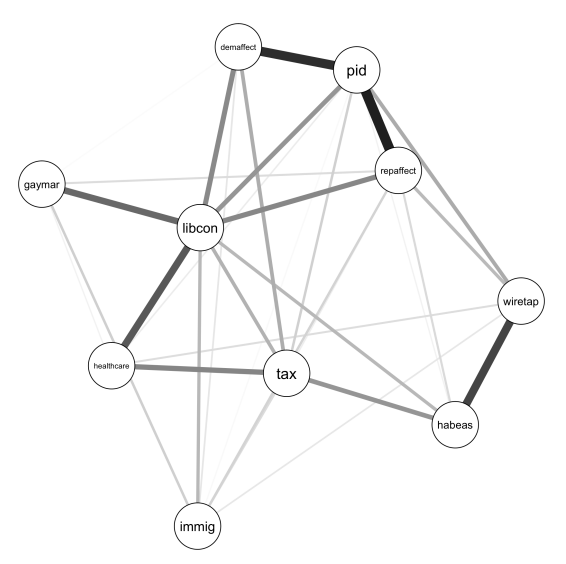

(c) High-knowledge, Wave 1

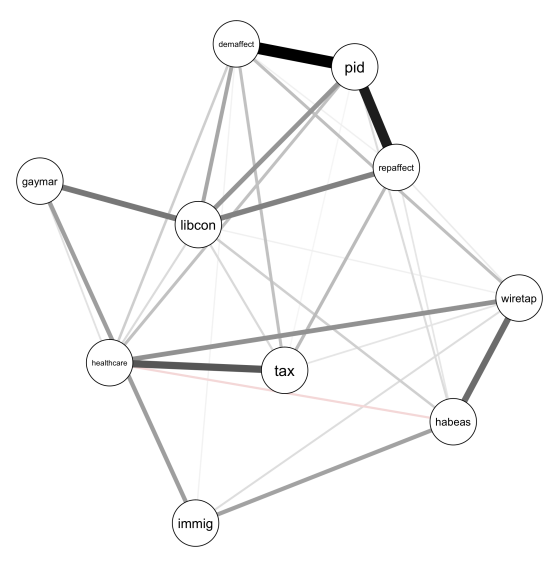

(b) Low-knowledge, Wave 10

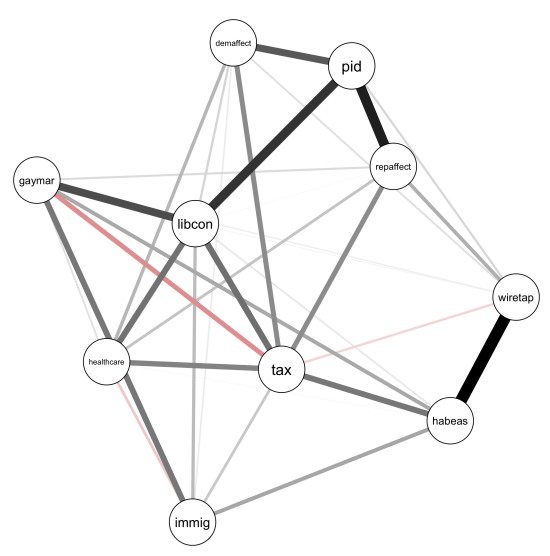

(d) High-knowledge, Wave 10

Figure 1: Belief Networks for high- and low-knowledge respondents at Waves 1 and 10. Note: Thicker edges indicate a weight with a higher absolute value. Node position is maintained across all four visualizations. 
Wave 1 Low Knowledge Strength Centrality Status 追 Operational $\oplus$ Symbolic

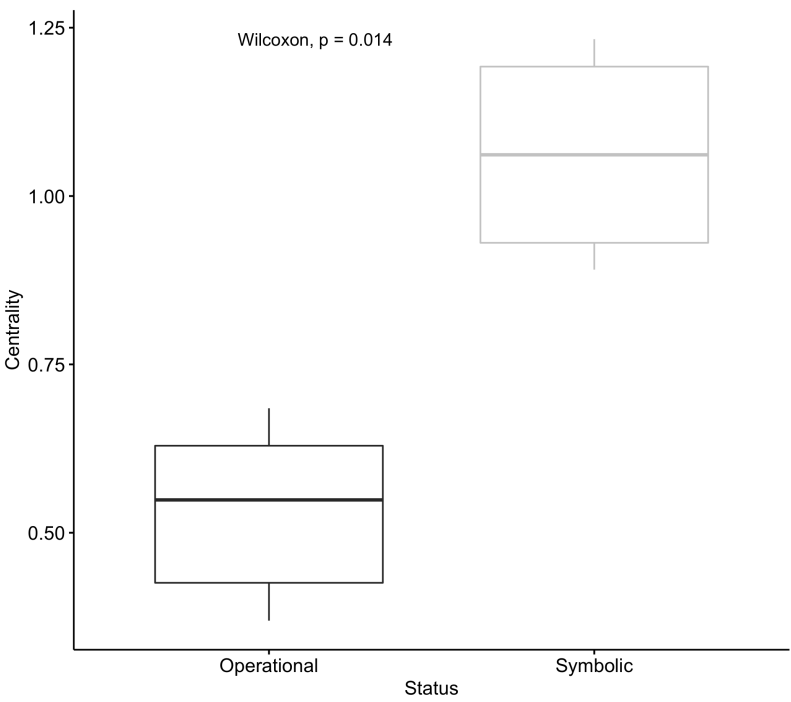

(a) Low knowledge, Wave 1

Wave 1 High Knowledge Strength Centrality

Status 追 Operational $\risingdotseq$ Symbolic

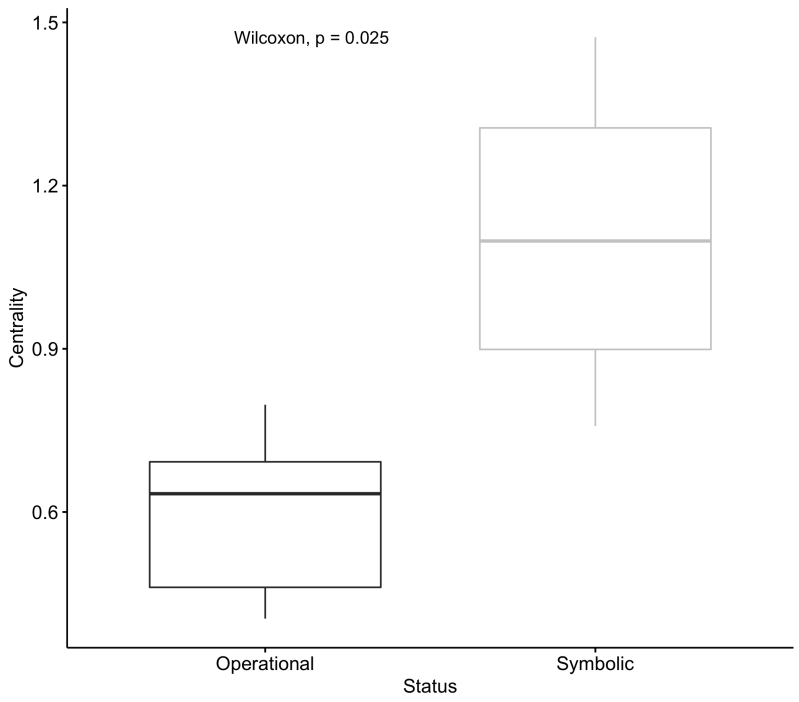

(c) High knowledge, Wave 1
Wave 10 Low Knowledge Strength Centrality

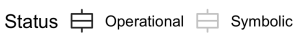

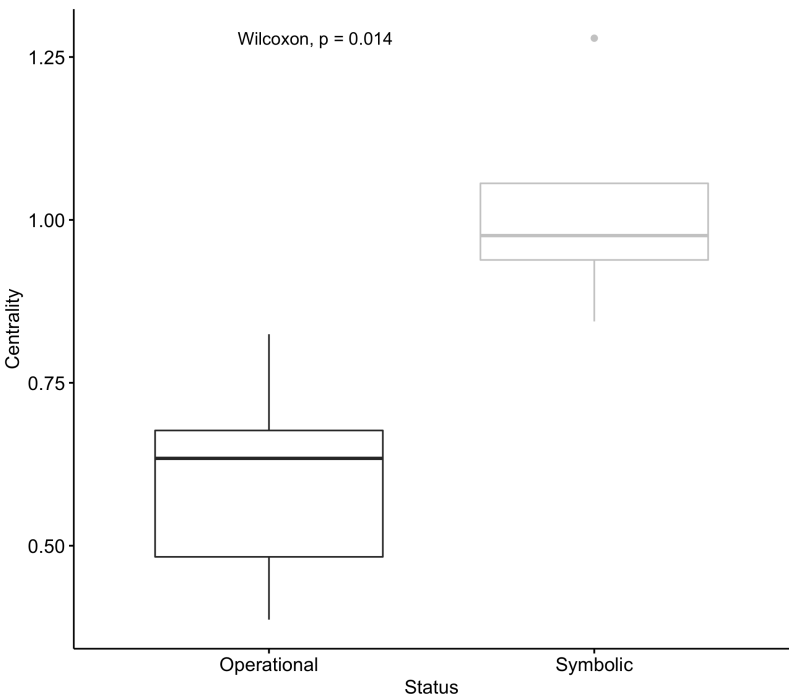

(b) Low knowledge, Wave 10

Wave 10 High Knowledge Strength Centrality Status 追 Operational $\risingdotseq$ Symbolic

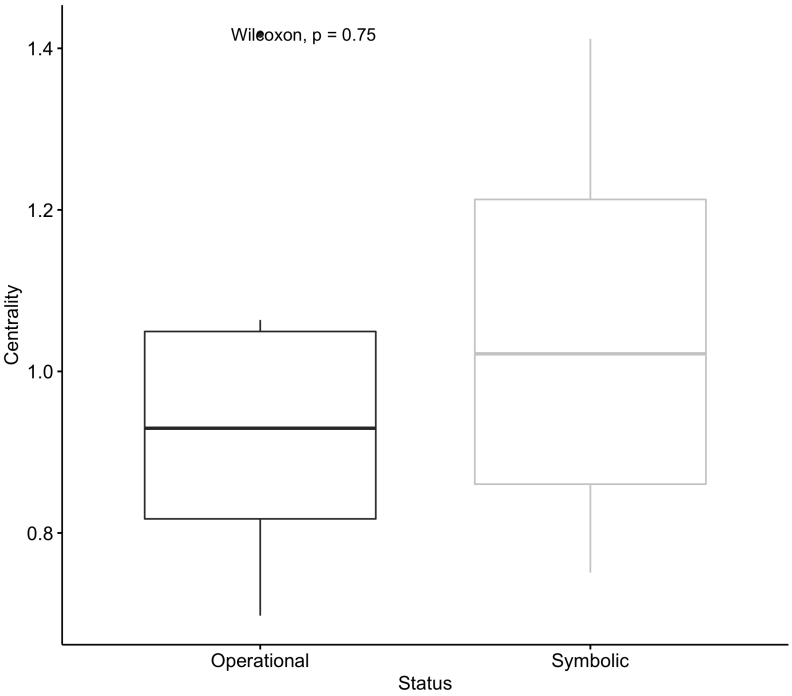

(d) High knowledge, Wave 10

Figure 2: Strength centrality estimates associated with operational and symbolic elements for highand low-knowledge respondents at Waves 1 and 10. Note: Box plots convey mean centrality among respective elements at given time step. 


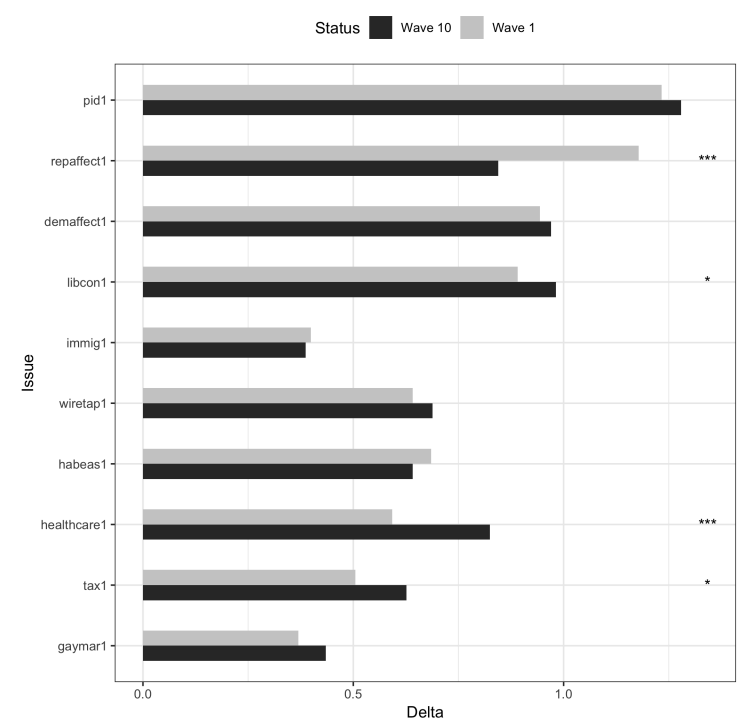

(a) Low-knowledge Strength Centrality

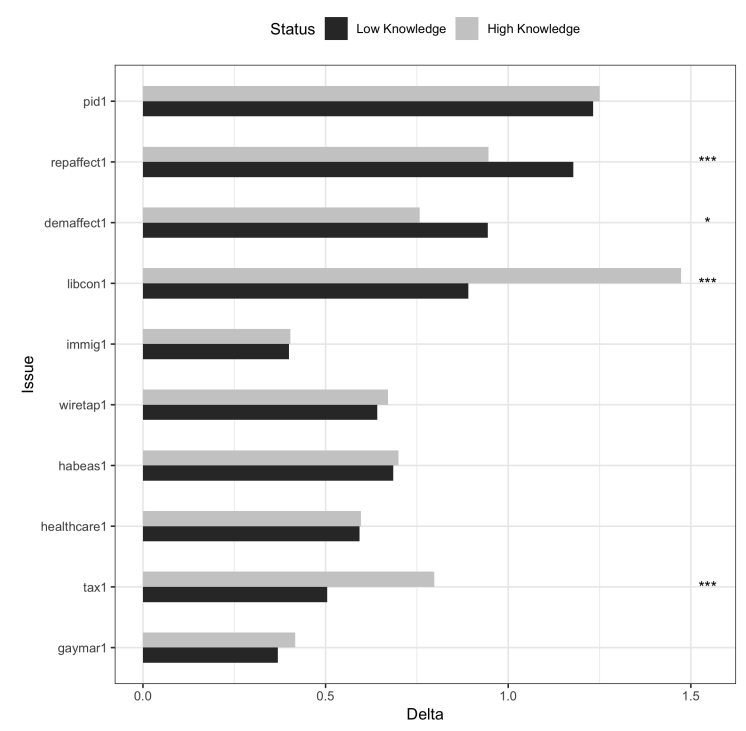

(c) Wave 1 Strength Centrality

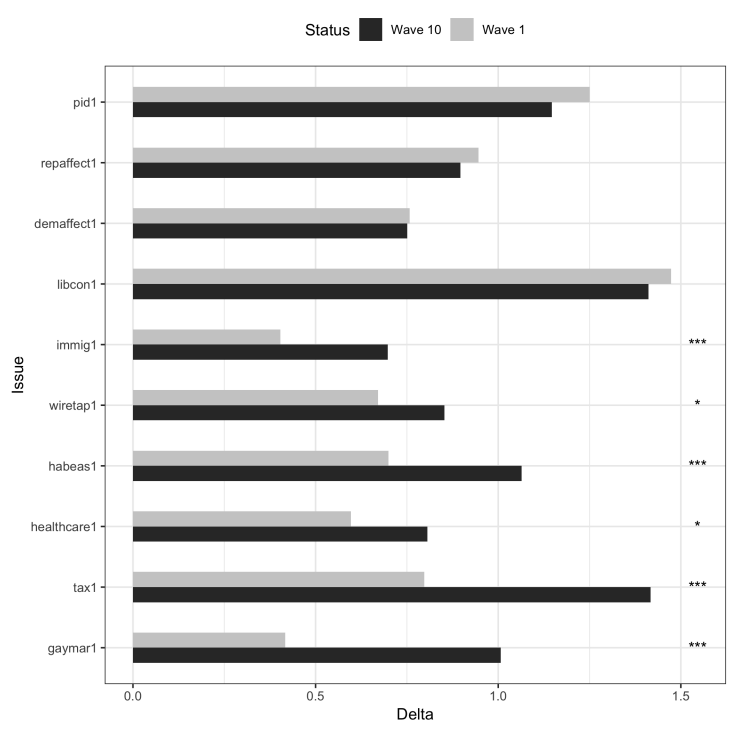

(b) High-knowledge Strength Centrality

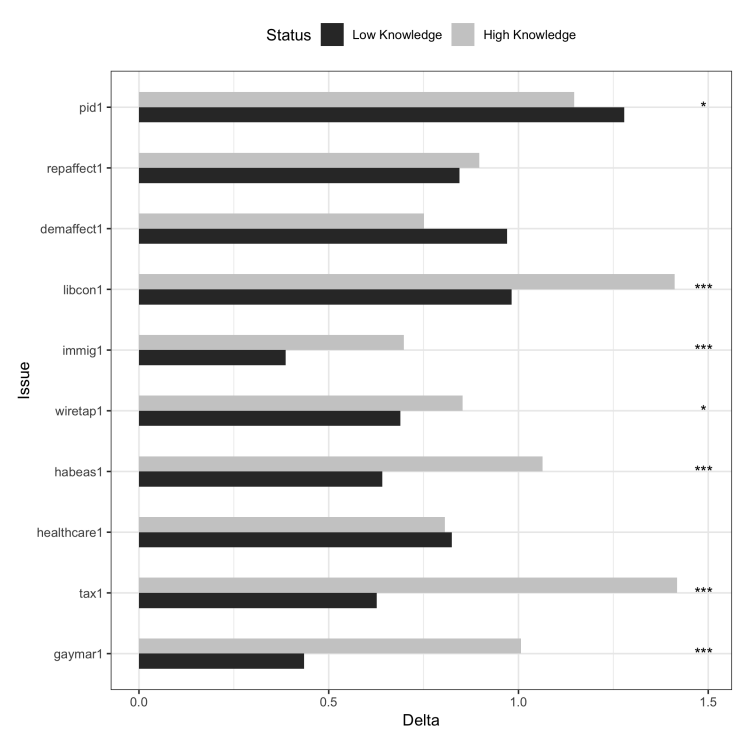

(d) Wave 10 Strength Centrality

Figure 3: Node strength centrality of high- and low-knowledge belief networks across waves. Note: Bars convey node strength centrality for respective item by group or wave. ${ }^{*} p<0.05,{ }^{* *} p<0.01,{ }^{* * *} p<0.001$ 


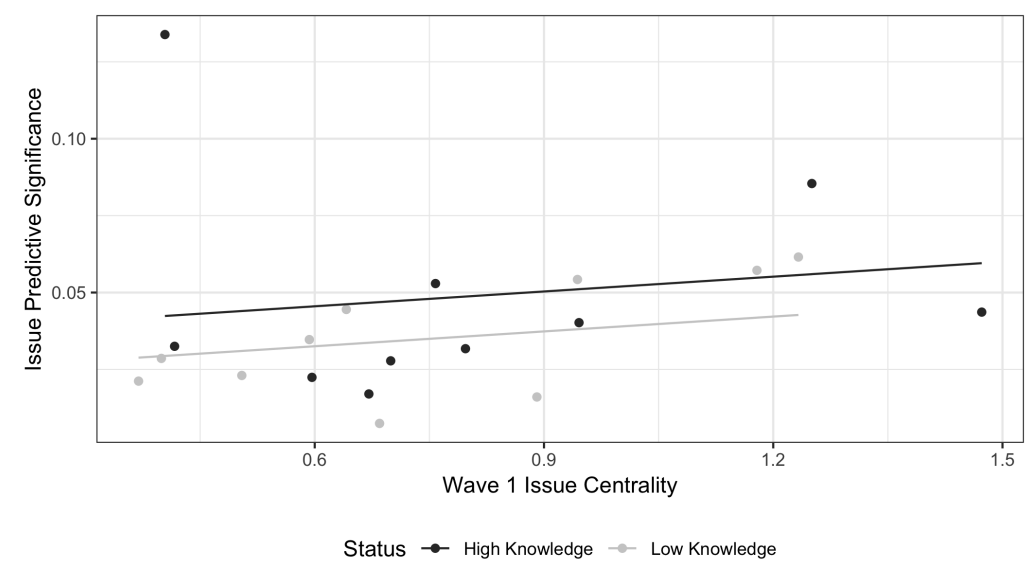

(a) Centrality to Predictive Significance

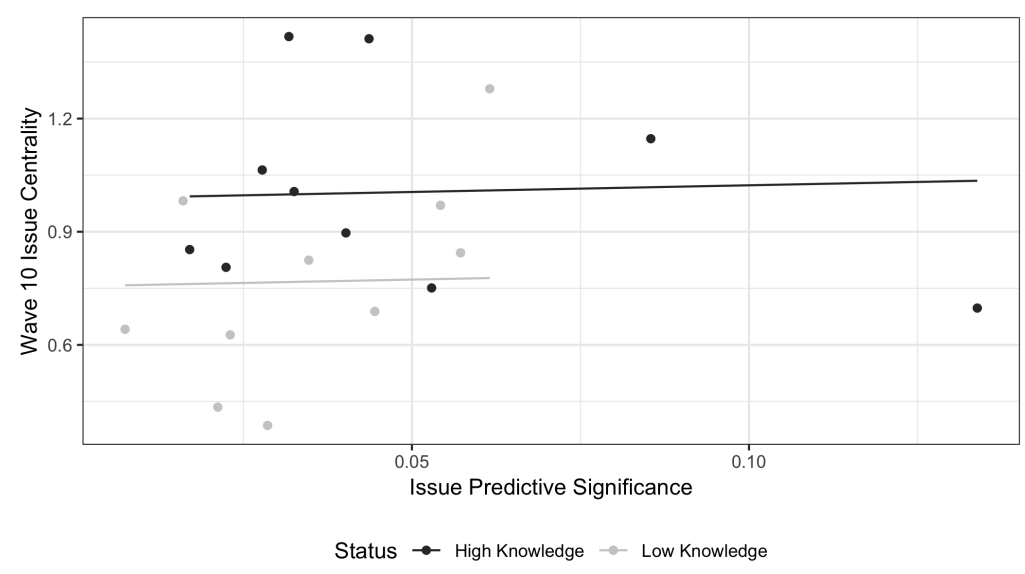

(b) Predictive Significance to Centrality

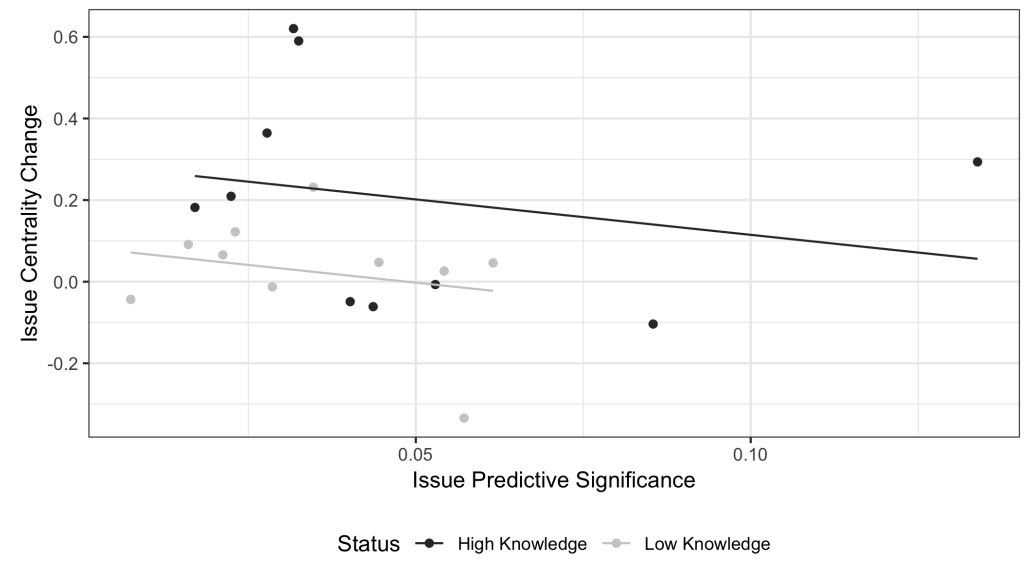

(c) Predictive Significance to Centrality Change

Figure 4: Panel models of the relationship between predictiveness and centrality. Note: Predictiveness is operationalized as the sum the variance that a given belief element predicts across other belief elements. Strength centrality for a given belief element conveys the sum of all absolute edge weights to which a node is directly connected. "Change" in predictiveness and centrality is derived by taking Wave 10 estimates less Wave 1. Underlying models include dummy variable for knowledge group, to which a linear fit has been superimposed. 